\title{
Para além da avaliação de qualidade
}

Heleno B., * Pinto D., *

E m Portugal, tem havido um interesse crescente em fazer investigação sobre qualidade nos cuidados de saúde primários. Prova disso é o aumento do número de trabalhos da área da qualidade, submetidos para publicação na Revista Portuguesa de Clínica Geral (RPCG) ou apresentados em congressos e reuniões de medicina geral e familiar. No entanto, a publicação de relatos de avaliação de qualidade ou de relatos de melhoria de qualidade tem-se revelado tão desafiante quanto problemática. Neste editorial, procura- se divulgar a política editorial da RPCG para a publicação de trabalhos na área da qualidade e comentar alguns problemas encontrados com frequência nos artigos recebidos.

Embora intimamente ligados, é útil distinguir os trabalhos de avaliação de qualidade dos trabalhos de garantia e melhoria de qualidade. Os primeiros são estudos pontuais. Neles procura-se identificar problemas na prestação de cuidados de saúde; compara-se os resultados obtidos com critérios de qualidade pré-definidos; e, por fim, propõe-se acções para a sua correcção. Já nos trabalhos de garantia e melhoria de qualidade, completa-se o ciclo da qualidade. Parte-se de uma avaliação prévia do problema, executa-se uma intervenção e volta-se a avaliar para saber se as medidas correctoras surtiram efeito.

Os estudos de qualidade que têm sido submetidos para publicação na RPCG são, na sua maioria, trabalhos de avaliação de qualidade. Alguns são realizados com um rigor metodológico indiscutível. Mas, uma vez que se limitam a fazer um diagnóstico de situação, raramente trazem conhecimento relevante para quem não pertence à(s) unidade(s) de saúde avaliadas. De alguma forma, os trabalhos de garantia e melhoria de qualidade são menos sensíveis à questão do contexto. Se houver uma boa descrição, quer das intervenções utilizadas, quer das características do local onde a inter-

*Editor da Revista Portuguesa de Clínica Geral. venção foi realizada, outros profissionais poderão julgar se a intervenção é transferível para o seu contexto de trabalho. ${ }^{2}$ Os trabalhos de garantia e melhoria de qualidade serão valorizados face aos de avaliação de qualidade.

Frequentemente encontramos trabalhos de qualidade cuja descrição metodológica se limita ao preenchimento de uma grelha de avaliação. Para assegurar que as intervenções de melhoria de qualidade são descritas de forma exaustiva e transparente, foram criadas as normas SQUIRE (Standards for QUality Improvement Reporting Excellence), ${ }^{3,4}$ adoptadas pela RPCG em $2009 .{ }^{5}$ A leitura destas recomendações poderá ajudar potenciais autores de trabalhos de melhoria de qualidade a incluir informação necessária para a caracterização da intervenção e caracterização do contexto. Apenas serão considerados para publicação trabalhos com descrição metodológica adequada.

Existem alguns problemas metodológicos recorrentes. É frequente a utilização de amostras de conveniência que não representam adequadamente a unidade em estudo, a comparação de proporções não corroborada pela utilização de testes estatísticos ou a aplicação de testes estatísticos sem cumprir as condições de aplicação. O estudo descritivo transversal parece ser ubíquo, mesmo quando outras metodologias pareçam mais úteis face aos objectivos que são propostos no trabalho. É preciso reconhecer que há críticas à robustez e validade dos trabalhos de avaliação de qualidade. ${ }^{6} \mathrm{De}$ forma a corroborar a força da prova deste tipo de trabalhos foi sugerida, por exemplo, a utilização simultânea de métodos quantitativos e qualitativos. ${ }^{7,8}$ Outra questão prende-se com a escolha da fonte de dados. Os trabalhos submetidos utilizam, quase exclusivamente, dados provenientes do processo clínico. É rara a utilização de outras fontes de dados, como a observação directa de comportamentos ou as entrevistas; quando estas permitiriam ter uma perspectiva mais completa do problema abordado. Na decisão de publicação, serão 
valorizados trabalhos que utilizem metodologias inovadoras.

Do ponto de vista formal, um indicador terá que ter, pelo menos, validade facial e ser estável, concreto e relevante. ${ }^{9}$ Garantir que um indicador tenha estas características é, em si mesmo, um trabalho de investigação exigente. Variáveis de resultado como a mortalidade ou o número de eventos têm estas quatro características; mas, em cuidados primários, são eventos raros, o que limita a sua utilização para comparar diferentes unidades de saúde ou a mesma unidade de saúde em diferentes momentos do tempo. Os resultados de ensaios clínicos recentes sobre controlo glicémico na diabetes vieram levantar dúvidas sobre a utilização de variáveis intermédias como a hemoglobina glicosilada. Variáveis orientadas para o doente como a satisfação, a incapacidade, a capacitação dos doentes têm uma importância extrema em cuidados primários mas são difíceis de medir. Dado que a validação de indicadores é crucial para a investigação em qualidade, este tipo de artigos será muito valorizado na decisão de publicação. A maioria dos autores poderá não se sentir confortável com as metodologias de validação dos indicadores. Uma estratégia para ultrapassar esta dificuldade é a utilização de indicadores desenvolvidos por grupos internacionais. ${ }^{10-16}$ No entanto, é preciso reconhecer que muitos destes indicadores foram desenvolvidos para a contratualização e não para a investigação. Caberá aos autores de trabalhos de qualidade reflectir se é razoável assumir que o indicador escolhido mede os aspectos de qualidade propostos nos objectivos do trabalho e se é relevante para a prática clínica.

Alguns dos nossos autores deparam-se com dificuldades no estabelecimento de critérios de qualidade. Por um lado, porque faltam estudos nacionais com os quais se possam comparar (critérios empíricos de qualidade); por outro, em cuidados primários existem vozes discordantes à utilização das metas propostas nas normas de orientação clínica (critérios normativos de qualidade). ${ }^{17,18}$ Perante estas dificuldades, é essencial que os autores discutam a validade do critério de qualidade que adoptam. Nesta discussão, é necessário ter atenção que, numa parte não desprezável dos utentes, os médicos poderão optar por não seguir as normas de orientação clínica por razões válidas, tornando a não adesão num processo consciente, fruto do seu juízo clí- nico. ${ }^{19-21}$ Por outro lado, os factores responsáveis pela não adesão poderão ser intransponíveis, a validade científica da norma pode ser questionada pelos médicos ou esta poderá não ser aplicável ao seu contexto. ${ }^{22}$ Nestes casos, a estratégia de melhoria de qualidade poderá passar pela aplicação de outra norma de orientação ou aperfeiçoamento da existente. Assim, sem um desenho de estudo metodologicamente apropriado para identificar as causas do não cumprimento de uma norma, não é válido assumir que tal se deve a desconhecimento dos médicos.

A identificação de quais os factores que concorrem para o resultado final é ainda essencial para que possa ser desenhada e implementada uma estratégia de melhoria da qualidade. A forma clássica - fornecer mais educação sobre o tema aos médicos - tem muito pouca eficácia ou pode mesmo não trazer qualquer benefício. ${ }^{23-25}$ De facto, nas normas de orientação que abordam as patologias mais frequentes na consulta do médico de família, a falta de conhecimentos não foi identificada como uma barreira significativa à implementação. ${ }^{22,26}$ Assim, a educação dos médicos sobre um tema parece ser um componente necessário, mas não suficiente para melhorar o seu desempenho. Esta transição afigura-se bem mais complexa, o que levou à conceptualização de modelos teóricos da passagem do conhecimento à acção, onde a avaliação dos problemas, adaptação às realidades locais e avaliação da sustentabilidade dos resultados são componentes-chave para a mudança. ${ }^{27}$ Os autores devem ter em conta as diversas estratégias possíveis para melhorar o desempenho dos médicos e serviços de saúde, evitando propor e implementar soluções que já foram anteriormente testadas sem sucesso.

A Revista Portuguesa de Clínica Geral gostaria de dar o seu contributo para que, na área da qualidade, se investigue mais e melhor. Nesse sentido, convidamos os autores de trabalhos de avaliação de qualidade a irem mais além e partilharem as suas aprendizagens após terem completado os ciclos de melhoria de qualidade. Para que outros possam ter ganhos ao ler o relato de melhoria de qualidade, reforçamos a necessidade de descrever detalhadamente o contexto e as características da intervenção. Para facilitar a apreciação da relevância do trabalho, desafiamos os autores a explicitarem a linha de raciocínio que levou à escolha do dese- 
nho de estudo, das fontes de dados, dos indicadores e dos critérios de qualidade. Por fim, devem ser identificadas as causas que tenham contribuído para os resultados observados e estas devem ser tidas em consideração na implementação de uma estratégia de melhoria contínua da qualidade e avaliação da sua eficácia.

\section{REFERÊNCIAS BIBLIOGRÁFICAS}

1. Puime A, Hernández P, Palomer R. Mejora de la calidad en Atención Primaria. In: Zurro AM, Pérez JF. Atencion primaria: conceptos, organizacion y practica clinica. Barcelona: Elsevier; 2008.

2. Thomson RG, Moss FM. QIR and SQUIRE: continuum of reporting guidelines for scholarly reports in healthcare improvement. Qual Saf Health Care 2008 Oct; 17 Suppl 1: i10-12.

3. Davidoff F, Batalden P, Stevens D, Ogrinc G, Mooney S. Publication guidelines for quality improvement in health care: evolution of the SQUIRE project. Qual Saf Health Care 2008 Oct; 17 Suppl 1: i3-9.

4. Ogrinc G, Mooney SE, Estrada C, Foster T, Goldmann D, Hall LW, et al. The SQUIRE (Standards for QUality Improvement Reporting Excellence) guidelines for quality improvement reporting: explanation and elaboration. Qual Saf Health Care 2008 Oct; 17 Suppl 1: i13-32.

5. Conselho Editorial da Revista Portuguesa de Clínica Geral. Normas para apresentação de artigos à Revista Portuguesa de Clínica Geral. Rev Port Clin Geral 2009 Jan-Fev ; 25 (1): 130-44.

6. Auerbach AD, Landefeld CS, Shojania KG. The tension between needing to improve care and knowing how to do it. N Engl J Med 2007 Aug 9; 357 (6): 608-13.

7. Brown C, Hofer T, Johal A, Thomson R, Nicholl J, Franklin BD, et al. An epistemology of patient safety research: a framework for study design and interpretation. Part 2. Study design. Qual Saf Health Care 2008 Jun; 17 (3): 163-9.

8. Brown C, Hofer T, Johal A, Thomson R, Nicholl J, Franklin BD, et al. An epistemology of patient safety research: a framework for study design and interpretation. Part 3. End points and measurement. Qual Saf Health Care 2008 Jun; 17 (3): 170-77.

9. Donabedian A. Evaluating the quality of medical care. 1966. Milbank Q 2005; 83 (4): 691-729.

10. Kerr E; Rand Corporation. Quality of care for cardiopulmonary conditions: a review of the literature and quality indicators. Santa Monica, CA: Rand Health; 2000.

11. McGlynn E; Rand Corporation. Quality of care for children and adolescents: a review of selected clinical conditions and quality indicators. Santa Monica, CA: Rand; 2000.

12. Kerr E; Rand Corporation. Quality of care for general medical conditions: a review of the literature and quality indicators. Santa Monica, CA: RAND Health; 2000.

13. Asch S; Rand Corporation. Quality of care for oncologic conditions and
HIV: a review of the literature and quality indicators. Santa Monica, CA: Rand; 2000.

14. McGlynn E; Rand Corporation. Quality of care for women: a review of selected clinical conditions and quality indicators. Santa Monica, CA: Rand; 2000.

15. Indicatoren [Internet]. [citado em 09/04/2009]. Disponível em: http://nhg.artsennet.nl/kenniscentrum/k_implementatie/Indicatoren_dub.htm

16. QOF Guidance 2009 [Internet]. [citado em 13/04/2010]. Disponível em: http://www.nhsemployers.org/Aboutus/Publications/Documents/QOF _Guidance_2009_final.pdf

17. Heath I, Rubinstein A, Stange KC, van Driel ML. Quality in primary health care: a multidimensional approach to complexity. BMJ 2009; 338: b1242.

18. Stange KC, Ferrer RL. The paradox of primary care. Ann Fam Med 2009 Aug; 7 (4): 293-9.

19. Cabana MD, Rand CS, Powe NR, Wu AW, Wilson MH, Abboud PA, et al. Why don't physicians follow clinical practice guidelines? A framework for improvement. JAMA 1999 Oct 20; 282 (15): 1458-65.

20. Francke AL, Smit MC, de Veer AJ, Mistiaen P. Factors influencing the implementation of clinical guidelines for health care professionals: a systematic meta-review. BMC Med Inform Decis Mak 2008; 8 (1): 38.

21. Ab E, Denig P, van Vliet T, Dekker JH. Reasons of general practitioners for not prescribing lipid-lowering medication to patients with diabetes: a qualitative study. BMC Fam Pract 2009; 10 (1): 24

22. Lugtenberg M, Zegers-van Schaick JM, Westert GP, Burgers JS. Why don't physicians adhere to guideline recommendations in practice? An analysis of barriers among Dutch general practitioners. Implementation Sci 2009; 4 (1): 54.

23. Thomson O'Brien MA, Freemantle N, Oxman AD, Wolf F, Davis DA, Herrin J. Continuing education meetings and workshops: effects on professional practice and health care outcomes. Cochrane Database Syst Rev 2001; (2): CD003030.

24. Green LA, Wyszewianski L, Lowery JC, Kowalski CP, Krein SL. An observational study of the effectiveness of practice guideline implementation strategies examined according to physicians' cognitive styles. Implementation Sci 2007; 2 (1): 41.

25. Davis DA, Taylor-Vaisey A. Translating guidelines into practice: a systematic review of theoretic concepts, practical experience and research evidence in the adoption of clinical practice guidelines. CMAJ 1997 Aug $15 ; 157$ (4): 408-16.

26. Heneghan C, Perera R, Mant D, Glasziou P. Hypertension guideline recommendations in general practice: awareness, agreement, adoption, and adherence. Br J Gen Pract 2007 Dec; 57 (545): 948-52.

27. Straus SE, Tetroe J, Graham I. Defining knowledge translation. CMAJ 2009 Aug 4; 181 (3-4): 165-8.

Os autores declaram não possuir quaisquer conflitos de interesses. 